\title{
Interactive comment on "Consistency of dimensional distributions and refractive indices of desert dust measured over Lampedusa with IASI radiances” by G. Liuzzi et al.
}

\section{Anonymous Referee \#1}

Received and published: 19 November 2016

In this study, the authors make use of airborne measurements made over the Western Mediterranean during the ADRIMED campaign, as well as a dataset of complex dust aerosol refractive indices obtained in the lab to test the capability of the newly developed S-IASI-as radiative transfer code to mimic the IASI spectra in the presence of dust advected from Africa over the sea. The study makes use of 3 days of measurements of ADRIMED, which are not presented (they are extensively covered in Denjean et al. 2016), used to constrain the S-IASI-as algorithm. The strengths and limitations of the $\mathrm{s}$-IASI-as algorithm are discussed on the basis of the comparison with real IASI spectra in the $700-2800 \mathrm{~cm}-1$ spectral range.

Overall, this is an interesting study which deserves publication in AMT. The quality 
of the English could be improved. Also, some relevant references are missing which should be included. Finally, the authors should to a more thorough job at quantifying the errors and uncertainties in the different part of the spectrum. Also the impact of these errors and uncertainties on the aerosols microphysical properties (as presented in Section 2.2) derived from s-IASI-as.

Based on the above, I recommended that the paper be published in AMT provide that the above mentioned comments as well as the ones below are taken into account.

Recommendation: minor revision

Abstract - Define ChArMEx - "a dust event which occurred": based on the following, you have not looked at just a single dust event - there is no such thing as the southern med basin: here you are referring to the southern part of the Western Med basin - lines 5-9: the sentence is too long and conveys more than one idea, rephrase

Introduction Page 1 - L15-16: there is also some work on the topic from Lars Kluser at DLR - L21-22: Note that profiling of dust was recently achieved over Eastern Asia by the group of Juan Cuesta in Creteil. Please cite:

J. Cuesta, M. Eremenko, C. Flamant, G. Dufour, B. Laurent, G. Bergametti, M. Höpfner, J. Orphal and D. Zhou, 2015: Satellite observation of the 3D distribution of dust plumes from IASI throughout a major desert dust outbreak across East Asia in March 2008, J. Geophys. Res., 120, 7099-7127, doi:10.1002/2014JD022406

Page 2 - L19: a high degree of generality - L20: the maximum fastness: lame, please rephrase - L27-28: complicated sentence: just write that ADRIMED was a component of ChArMEx SOP1 - L29: is the ATR-42 an aircraft? Who is the operator, SAFIRE?? Please provide some details. - L33: later ( $p$ 10) you write that on 22 June some of the dust is coming from Southern Morocco, which is not the Sahara.. please correct.

Data and methods Page 9 - L14-15: could you specify the number of IASI pixels in the box? What is the time of the IASI overpasses on the 3 days? - L16: the 3 days are 
characterized by a decreasing atmospheric dust load: unclear, please rephrase. - L18: here and elsewhere: should this be IFOV? - L27: is this wind speed at $10 \mathrm{~m}$ above AMTD sea surface? How does 10-m wind speed relate to surface sea surface emissivity? In case of strong winds, whitecaps develop at the sea surface that will modify significantly the surface emissivity. It would be worth checking what the sea state and surface wind speeds are around Lampedusa during the 3 days selected.

Interactive comment

Conclusions P13 - L30: cases rather than scenarios P14 - L9-10: unclear sentence, please rephrase

Interactive comment on Atmos. Meas. Tech. Discuss., doi:10.5194/amt-2016-256, 2016. 